\title{
Staying positive: going beyond Lindblad with perturbative master equations
}

\author{
Robert S. Whitney \\ Institut Laue-Langevin, 6 rue Jules Horowitz, B.P. 156, 38042 Grenoble, France.
}

\begin{abstract}
The perturbative master equation (Bloch-Redfield) is extensively used to study dissipative quantum mechanics — particularly for qubits despite the 25 year old criticism that it violates positivity (generating negative probabilities). We take an arbitrary system coupled to an environment containing many degrees-of-freedom, and cast its perturbative master equation (derived from a perturbative treatment of Nakajima-Zwanzig or Schoeller-Schön equations) in the form of a Lindblad master equation. We find that the equation's parameters are time-dependent. This time-dependence is rarely accounted for, and invalidates Lindblad's dynamical semigroup analysis. We analyze one such Bloch-Redfield master equation (for a two-level system coupled to an environment with a short but non-vanishing memory time), which apparently violates positivity. We show analytically that, once the time-dependence of the parameters is accounted for, positivity is preserved.
\end{abstract}

Keywords: dissipative quantum mechanics, decoherence, master equation, Bloch-Redfield, Lindblad, positivity, two-level system, qubit 


\section{Introduction}

No system is truly isolated from its environment, thus all quantum systems experience some amount of dissipation and decoherence [1, 2]. To understand the properties of real quantum systems we must understand the effect of dissipation in quantum mechanics. This is extremely relevant to recent works on qubits and quantum information processing (quantum computing and communication). In experiments [3, 4, 5] the coupling to the environment is typically not as small as would be required to build a quantum computer. One must understand the effect of the environment on a qubit, if one wishes to minimize it.

Any theory for a quantum system which exchanges energy and information (but not particles) with its environment should give a master equation (evolution equation) for the system's density-matrix which satisfies three basic requirements;

(i) preserves the Hermiticity of the density-matrix, so all probabilities are real,

(ii) preserves the trace of the density-matrix, then the sum of probabilities over any complete set of orthogonal states is one,

(iii) preserves positivity. A system is positive only if the probability of all possible states is positive. Given (ii), this guarantees that all probabilities lie between zero and one. In this work we do not consider complete positivity, excepting comments in Sections 2 and 7.

There are only a small number of models for which such master equations can be derived exactly (we will not address these here). In all other cases, there are two main methods for finding such a master equation [2];

- Phenomenological method. Here one attempts to construct general master equations which satisfy requirements (i-iii). Under the assumption that the evolution is translationally invariant in time (a dynamical semigroup property), as is often the case for Markovian evolution, Lindblad [7, 8] considered the master equation given in Eqs. $(1 a \mid 1 b)$. He proved that it is the most general equation that satisfies (i-ii) above, while also preserving complete positivity. Complete positivity is as strong or stronger than positivity, thus it automatically satisfies (iii) above (see the comment in Section 7 due to [6]).

- Perturbative method [1. Here one takes the evolution of a system and its environment (from their combined Hamiltonian), and traces over the environment degrees-of-freedom. Various methods of doing this exist; Bloch-Redfield $[$, 10, Nakajima-Zwanzig [11, 12, Schoeller-Schön[13]. However one is typically forced to treats the system-environment interaction perturbatively, then all these approaches reduce to Bloch-Redfield's.

The Lindblad master equation (the most general generator of a dynamical semigroup) takes the form;

$$
\begin{array}{r}
\frac{\mathrm{d}}{\mathrm{d} t} \hat{\rho}(t)=-\mathrm{i}\left[\mathcal{H}_{\mathrm{sys}}, \hat{\rho}(t)\right]_{-}-\sum_{n=1}^{N^{2}-1} \frac{\lambda_{n}}{2}\left(\hat{L}_{n}^{\dagger} \hat{L}_{n} \hat{\rho}(t)+\hat{\rho}(t) \hat{L}_{n}^{\dagger} \hat{L}_{n}-2 \hat{L}_{n} \hat{\rho}(t) \hat{L}_{n}^{\dagger}\right), \\
\text { with } \lambda_{n} \geq 0 \text { for all } n,
\end{array}
$$

where the commutator $[\hat{A}, \hat{B}]_{-}=\hat{A} \hat{B}-\hat{B} \hat{A}$, and $\left\{\hat{L}_{n}\right\}$ is a set of ortho-normal (traceclass) operators. It is often assumed that all Markovian master equations fall into the category of dynamical semigroup evolution, and thus Eqs. $1 a \mid 1 b$ give the most 


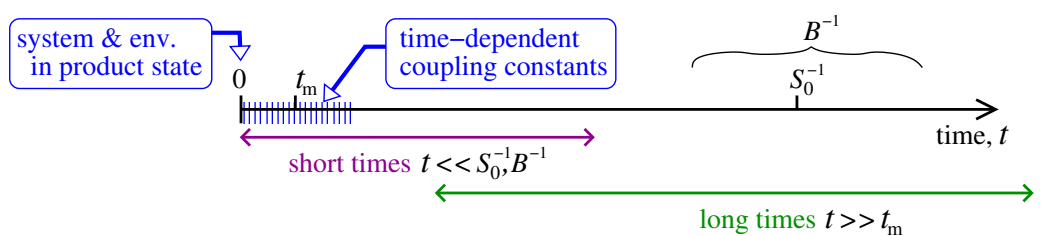

Figure 1. Timescales for the model (in Section 6 for which we show positivity, despite it not satisfying Lindblad's requirement, Eq. $1 b$. The system is a spinhalf with Hamiltonian $-\frac{1}{2} B \hat{\sigma}_{z}$, and the environment couples to it via $\hat{\sigma}_{x}$. The environment's noise spectrum (with noise power $S_{0}$ ) is broad, leading to a short memory time, $t_{\mathrm{m}}$. Decoherence and relaxation times (both $\sim S_{0}^{-1}$ ) can be smaller or larger than the Larmor precession period, $B^{-1}$.

general Markovian evolution. However this is a subtle point, we discuss it (and define terms like "dynamical semigroup" and "trace-class") in Section 2

The perturbative method's advantage over the phenomenological method is that one can study how a particular environment (with a given spectrum, temperature, etc) affects the system. Thus one can address a crucial aspect of qubit research; how should one engineer a particular system to minimize decoherence? However the resulting Bloch-Redfield master equation has long been criticized [14, 15], because it can be written in the form in Eq. (1a) but then typically violates Eq. (1b). In these cases it violates Lindblad's condition for complete positivity. Further, there is plenty of evidence that it also violates positivity (see Section 1.2).

\subsection{Outline of this article}

The objective of this article is to study this apparent contradiction between the perturbative method and Lindblad's proof. We start by discussing, in Section 2 . the assumptions that underlie the Lindblad master equation. In Section 3 we consider the Bloch-Redfield equation for an arbitrary system, and show that, in general, one coupling constant, $\lambda_{2}$, is negative. However we also show that the parameters of the Bloch-Redfield master equation, $\left\{\lambda_{n}\right\}$ and $\left\{\hat{L}_{n}\right\}$, are time-dependent. This means the master equation does not generate a dynamical semigroup. Thus Lindblad's proof is inapplicable to the Bloch-Redfield equation, and a priori we do not know whether a negative $\lambda_{2}$ will lead to a violation of positivity or not.

In Section 6, we consider the Bloch-Redfield equation for a particular system (a two-level system coupled to an environment with a very broad spectrum of excitations). We divide the evolution into two overlapping regimes; short- and longtimes (sketched in Fig. 11). The time-dependence of the parameters is only relevant in the short-time regime ( $t$ much less than decoherence/relaxation times). We show analytically that the system remains positive in both regimes (i.e. for all $t \geq 0$ ), despite the negative coupling constant, $\lambda_{2}$.

\subsection{The place of this work in the literature}

In traditional derivations of the Bloch-Redfield master equation [1, 2], it is assumed that the parameters of the master equation are time-independent. In reality all environment-induced terms in the master equation are zero at $t=0$ (defined as the time at which the system and environment are in a factorized state), before growing with $t$ and saturating at $t \gg t_{\mathrm{m}}$, where $t_{\mathrm{m}}$ is the environment memory time. So the 
assumption of time-independence is flawed for times of order the memory time, $t_{\mathrm{m}}$. This has been discussed in the context of coupled classical oscillators [16, over-damped Brownian motion [17, damped quantum oscillators [18, 19, 20, 21, 22], dissipative two-level systems [23, 24, 20, 25] and more generally [26, 27]. Nearly all these works consider dynamics on times of order the $t_{\mathrm{m}}$ as an initial-slip, after which the dynamics is given by the time-independent master equation, the justification for this is sketched in Appendix B. Of most relevance to us are those works which try to show that positivity is preserved in this context 22, 24, 20, 21, 25, 27. However these works provide only plausibility arguments [28, or numerical studies (they evolved a finite number of initial conditions and checked that negative probabilities did not emerge). In contrast, for our model, we consider all possible initial conditions and thereby prove analytically that positivity is preserved.

It has been noted that course-graining can ensure positivity [29. The work presented here indicates that the usual assumption of time-independent parameters in the master equation only leads to a violation of positivity for $t \lesssim t_{\mathrm{m}}$. Thus coursegraining on such a scale could hide such a violation. It is also common to simplify Bloch-Redfield equations by making a rotating-wave approximation [14, 15, 30, which is also a form of course-graining since it "averages out" fast oscillations. However if we treat the time-dependence of the parameters correctly, the Bloch-Redfield equation is derived without any approximations which fail on short timescales, so it should preserve positivity without any course-graining.

There has been a lot of interest in a particular class of non-Markovian master equations which are positive by construction. They are either constructed by averaging Markovian master equations [31, or by measurement processes [32. However, while these models are extremely interesting, we are not aware of works relating them to microscopic models of a typical qubit experiencing dissipation 33.

Finally, we mention that some works suggested that the reason for negative probabilities was the choice of factorized initial conditions 30, 34. They argued that this initial condition was unphysical, and a more physical initial condition would not generate negative probabilities. However, factorized initial conditions correspond to any situation in which one makes a projective measurement of the system state at the start of the evolution. Thus, while other initial conditions are worthy of study [35, 36] (and highly relevant to certain experimental protocols), a factorized initial condition is not unphysical, and thus should not be able to generate negative probabilities. In this work we restrict ourselves to factorized initial conditions (see Section 3).

\section{The Lindblad master equation}

The Lindblad master equation, Eqs. $1 a \mid 1 b)$, is written in terms of a set of $N^{2}$ traceclass operators, $\left\{\hat{L}_{n}\right\}$ (where $N$ is the number of levels of the system). Operators are trace-class if they form a complete orthonormal basis in the space of system operators, with the scalar-product defined as $\left(\hat{L}_{i}^{\dagger} \cdot \hat{L}_{j}\right) \equiv \operatorname{tr}\left[\hat{L}_{i}^{\dagger} \hat{L}_{j}\right]$, see [37. The basis is complete

if any system operator can be written as $\hat{O}_{\text {sys }}=\sum_{j} \hat{L}_{j} \operatorname{tr}\left[\hat{L}_{j}^{\dagger} \hat{O}_{\text {sys }}\right]$. We choose $L_{0}$ to be proportional to the unit matrix. One can see that Eq. (1a) preserves the Hermiticity and trace of the system's density-matrix (the latter requires cyclic permutations inside the trace). The combination of Eq. (1b) with Eq. (1a) guarantees positivity. In fact it guarantees a stronger condition called complete positivity, which is the requirement that all probabilities remain positive even if the system became entangled with a second system at $t<0$, but then does not interact with it again. For a review see 
Sections 2.4 and 3 of Ref. [2], section VB of Ref. [15] or the introduction of Ref. [36]. In this article we concern ourselves with studying positivity not complete positivity, however it has recently been shown [ [6] that the two are equivalent for the model that we study in section 6 .

Lindblad proved that Eqs. $1 a \mid 1 b)$ give the most general dynamical semigroup evolution [7, 8. However to understand if this is applicable to a given system, one must ask if that system has the properties of a dynamical semigroup. For this one looks at the density-matrix propagator $\mathbb{K}\left(t ; t_{0}\right)$, which acts on the density-matrix at $t_{0}$ to give the density-matrix at time $t$, so in terms of matrix elements

$$
\hat{\rho}_{i^{\prime} j^{\prime}}(t)=\sum_{i j} \mathbb{K}_{i^{\prime} j^{\prime} ; i j}\left(t ; t_{0}\right) \hat{\rho}_{i j}\left(t_{0}\right)
$$

This super-operator, $\mathbb{K}\left(t ; t_{0}\right)$, is an $N \times N \times N \times N$ tensor which acts on the $N \times N$ density-matrix. Substituting it into Eq. (1a) gives a master equation for $\mathbb{K}_{i^{\prime} j^{\prime} ; i j}(t)$. The requirements for $\mathbb{K}\left(t ; t_{0}\right)$ to form a dynamical semigroup are given in Refs. 2, 8, 15, they include (i-ii) above and complete positivity. However another crucial requirement is that the propagator must be translationally invariant in time, so $\mathbb{K}\left(t ; t_{0}\right)=\mathbb{K}\left(t-t_{0}\right)$ for all $t, t_{0}>0$ (where the system and environment were in a factorized state at time $t=0)$. Only then does $\mathbb{K}_{i^{\prime \prime} j^{\prime \prime} ; i j}\left(t_{2}+t_{1}\right)=$ $\sum_{i^{\prime} j^{\prime}} \mathbb{K}_{i^{\prime \prime} j^{\prime \prime} ; i^{\prime} j^{\prime}}\left(t_{2}\right) \mathbb{K}_{i^{\prime} j^{\prime}: i j}\left(t_{1}\right)$. Thus a master equation must have time-independent parameters to have this semigroup property. If either the system Hamiltonian or the environment couplings (coupling constants $\lambda_{n}$ or operators $\hat{L}_{n}$ ) are time-dependent, then $\mathbb{K}\left(t ; t_{0}\right)$ is not translationally invariant in time. Thus Lindblad's proof is inapplicable for such systems, even if their evolution is Markovian (in the sense that $\mathrm{d} \hat{\rho}(t) / \mathrm{d} t$ is a function only of $\hat{\rho}(t)$ not $\left.\hat{\rho}\left(t^{\prime}<t\right)\right)$. So if $\lambda_{n}$ or $\hat{L}_{n}$ are time-dependent (as in our perturbative analysis) one cannot a priori state that negative $\lambda_{n}$ will lead to a violation of positivity.

\section{The perturbative (Bloch-Redfield) master equation}

We assume that the system and environment start (at $t=0$ ) in a factorized state $\hat{\rho}(t=0) \otimes \hat{\rho}_{\text {env }}$. This would be the case if the experiment started with a perfect projective measurement of the state of the system [38. The "universe" (system + environment) then evolves under the Hamiltonian,

$$
\hat{\mathcal{H}}_{\text {univ }}=\hat{\mathcal{H}}_{\text {sys }}+\hat{\mathcal{H}}_{\text {env }}+\hat{\Gamma} \hat{x}
$$

where $\hat{\Gamma}$ and $\hat{x}$ are system and environment operators, respectively. We treat these operators as Hermitian, because we assume they are observables (i.e. charge, magnetic dipoles, etc) as is the case in most qubit experiments (and more generally). Without lose of generality we can assume $\hat{\Gamma}$ is dimensionless and $\hat{x}$ has units of energy.

For a suitable environment one can derive the Bloch-Redfield master equation for the evolution of the system's reduced density-matrix, $\hat{\rho}(t)$, from the evolution of the universe's state (tracing out the environment at time $t$ ). The assumptions necessary to derive this master equation are discussed in Appendix A. Broadly speaking one needs an environment with a broad (almost) continuous spectrum of excitations, then the memory kernel of the environment (defined in Eq. (4c) below) decays on a timescale $t_{\mathrm{m}}$. Typically the Bloch-Redfield master equation is valid when the memory time, $t_{\mathrm{m}}$, is much less than timescales associated with dissipation (relaxation and decoherence), 
which go like $1 /\left(|\hat{\Gamma} \hat{x}|^{2} t_{\mathrm{m}}\right)$ (we set $\hbar=1$ throughout this article). The Bloch-Redfield master equation can be written as

$\frac{\mathrm{d}}{\mathrm{d} t} \hat{\rho}(t)=-\mathrm{i}\left[\mathcal{H}_{\mathrm{sys}}, \hat{\rho}(t)\right]_{-}-\hat{\Gamma} \hat{\Xi} \hat{\rho}(t)-\hat{\rho}(t) \hat{\Xi}^{\dagger} \hat{\Gamma}+\hat{\Xi} \hat{\rho}(t) \hat{\Gamma}+\hat{\Gamma} \hat{\rho}(t) \hat{\Xi}^{\dagger}$,

with $\Gamma$ being the operator in Eq. (3) and

$$
\hat{\Xi}=\int_{0}^{t} \mathrm{~d} \tau \alpha(\tau) \exp \left[-\mathrm{i} \hat{\mathcal{H}}_{\mathrm{sys}} \tau\right] \hat{\Gamma} \exp \left[\mathrm{i} \hat{\mathcal{H}}_{\mathrm{sys}} \tau\right]
$$

Unlike many derivations we do not assume that we can take the upper-bound on this integral to $\infty$. The function $\alpha(\tau)$ is the environment's memory kernel, given by

$$
\alpha(\tau)=\operatorname{tr}_{\text {env }}\left[\hat{x} \exp \left[-\mathrm{i} \hat{\mathcal{H}}_{\mathrm{env}} \tau\right] \hat{x} \exp \left[\mathrm{i} \hat{\mathcal{H}}_{\mathrm{env}} \tau\right] \hat{\rho}_{\text {env }}(t)\right] .
$$

Since $\alpha(\tau)$ is typically complex, $\hat{\Xi}$ is not usually Hermitian (unlike $\hat{\Gamma}$ ). We assume that $\alpha(\tau)$ is independent of $t$, then $\alpha(-\tau)=\alpha^{*}(\tau)$. This is true if the environment is large enough that it is unaffected by the system-environment coupling (during the experiment), and the initial environment state obeys $\left[\mathcal{H}_{\text {env }}, \hat{\rho}_{\text {env }}\right]=0$. The latter is the case if the environment is in an eigenstate or a classical mixture of eigenstates (such as a thermal state). We assume that $\alpha(\tau)$ is a decaying function of $\tau$, and define the memory time, $t_{\mathrm{m}}$, as the timescale of that decay. Then $\hat{\Xi}$ is $t$-dependent, because $t$ appears in the upper-bound on the integral in Eq. $4 b$.

Eq. (4a) looks Markovian, in the sense that the rate of change of $\hat{\rho}(t)$ depends only on the value of $\hat{\rho}(t)$ (not the value of $\hat{\rho}\left(t^{\prime}\right)$ for $t^{\prime}<t$ ). Despite this memory effects are present in the memory kernel, $\alpha(\tau)$. As we see in Appendix A if $\alpha(\tau)$ is finite for a given $\tau$ it means the rate of change of $\hat{\rho}$ at time $t$ is affected by $\hat{\rho}(t-\tau)$. This is the reason for the time-dependence of $\hat{\Xi}$, which is zero at $t=0$, and grows to saturate on a timescale of order the environment memory time, $t_{\mathrm{m}}$.

By writing $\hat{\Gamma} \hat{\Xi} \hat{\rho}-\hat{\rho} \hat{\Xi}^{\dagger} \hat{\Gamma}=\frac{1}{2}\left[\left(\hat{\Gamma} \hat{\Xi}+\hat{\Xi}^{\dagger} \hat{\Gamma}\right), \hat{\rho}\right]_{+}-\mathrm{i}\left[\frac{\mathrm{i}}{2}\left(\hat{\Gamma} \hat{\Xi}-\hat{\Xi}^{\dagger} \hat{\Gamma}\right), \hat{\rho}\right]_{-}$, where $[A, B]_{ \pm}=$ $A B \pm B A$ are the anti-commutator/commutator, Eq. (4a) becomes

$\frac{\mathrm{d}}{\mathrm{d} t} \hat{\rho}(t)=-\mathrm{i}\left[\hat{\mathcal{H}}_{\mathrm{sys}}^{\prime}, \hat{\rho}(t)\right]_{-}-\frac{1}{2}\left[\left(\hat{\Gamma} \hat{\Xi}+\hat{\Xi}^{\dagger} \hat{\Gamma}\right), \hat{\rho}(t)\right]_{+}+\hat{\Xi} \hat{\rho}(t) \hat{\Gamma}+\hat{\Gamma} \hat{\rho}(t) \hat{\Xi}^{\dagger}$,

where we define $\hat{\mathcal{H}}_{\text {sys }}^{\prime} \equiv \hat{\mathcal{H}}_{\text {sys }}-\frac{1}{2} \mathrm{i}\left(\hat{\Gamma} \hat{\Xi}-\hat{\Xi}^{\dagger} \hat{\Gamma}\right)$. Even when $\hat{\Xi} \neq \hat{\Xi}^{\dagger}$, both $\left(\hat{\Gamma} \hat{\Xi}+\hat{\Xi}^{\dagger} \hat{\Gamma}\right)$ and $\mathrm{i}\left(\hat{\Gamma} \hat{\Xi}-\hat{\Xi}^{\dagger} \hat{\Gamma}\right)$ are Hermitian. The fact that $\hat{\mathcal{H}}_{\text {sys }}^{\prime}$ is Hermitian means that we can interprete it as a renormalized system Hamiltonian.

It is very convenient to define the symmetrized and anti-symmetrized spectral function of the noise, $S(\omega)$ and $A(\omega)$ such that

$$
\begin{aligned}
& \frac{1}{2}[\alpha(\tau)+\alpha(-\tau)]=\operatorname{Re}[\alpha(\tau)]=\int \frac{\mathrm{d} \omega}{2 \pi} S(\omega) \exp [-\mathrm{i} \omega \tau], \\
& \frac{1}{2}[\alpha(\tau)-\alpha(-\tau)]=\mathrm{i} \operatorname{Im}[\alpha(\tau)]=\int \frac{\mathrm{d} \omega}{2 \pi} A(\omega) \exp [-\mathrm{i} \omega \tau],
\end{aligned}
$$

remembering that we set $\hbar=1$ throughout. One can extract the form of $S(\omega)$ and $A(\omega)$ from environment details (a bath of harmonic oscillators [39], a bath of spins [40], etc). For an environment in thermal equilibrium at temperature $T$ [41, $S(\omega)$ and $A(\omega)$ are related via $A(\omega)=S(\omega) \tanh \left(\omega / 2 k_{\mathrm{B}} T\right)$ [2]. For harmonic oscillators, $A(\omega) \propto J(\omega)$ and so $S(\omega) \propto J(\omega) \operatorname{coth}\left(\omega / 2 k_{\mathrm{B}} T\right)$, where $J(\omega)$ is the spectral-density in Ref. [39]. 


\subsection{Dephasing and Lamb shift when a rotating-wave approximation is reasonable}

When the dynamics is dominated by the system Hamiltonian (off-diagonal matrix elements decay over many Larmor oscillations), then we can make a rotating-wave (or secular) approximation 43 of Eq. (5). We write $\hat{\rho}(t)$ in the eigenbasis $\mathcal{H}_{\text {sys }}$ (so $\left.\mathcal{H}_{\text {sys } ; i j}=E_{i} \delta_{i j}\right)$, then we can expect $\hat{\rho}_{i j}^{\text {rot }}(t)=\mathrm{e}^{\mathrm{i}\left(E_{i}-E_{j}\right) t} \hat{\rho}_{\text {sys } ; i j}(t)$ to be insensitive to all fast oscillating contributions to its dynamics. We neglect ("average out") contributions to $\left(\mathrm{d} \hat{\rho}_{i j}^{\text {rot }} / \mathrm{d} t\right)$ which come from $\hat{\rho}_{\mathrm{sys} ; i^{\prime} j^{\prime}}$ when $i^{\prime} \neq i$ or $j^{\prime} \neq j$, since these contributions oscillate fast, at a rate $\left(E_{i^{\prime}}-E_{j^{\prime}}-E_{i}+E_{j}\right)$ 44. Then $(\mathrm{d} / \mathrm{d} t) \hat{\rho}_{i j}^{\text {rot }}=\left[\mathrm{i} \Delta E(i, j)-T_{2}^{-1}(i, j)\right] \hat{\rho}_{i j}^{\text {rot }}$. The dephasing rate, at which a super-position of states $i$ and $j$ decays to a classical mixture (1/ $T_{2}$ for two-level systems) is

$T_{2}^{-1}(i, j) \simeq \operatorname{Re}\left[\frac{1}{2}\left(\hat{\Gamma} \hat{\Xi}+\hat{\Xi}^{\dagger} \hat{\Gamma}\right)_{i i}+\frac{1}{2}\left(\hat{\Gamma} \hat{\Xi}+\hat{\Xi}^{\dagger} \hat{\Gamma}\right)_{j j}-\hat{\Xi}_{i i} \hat{\Gamma}_{j j}-\hat{\Gamma}_{i i} \hat{\Xi}_{j j}^{\dagger}\right]$.

The coupling to the environment also causes a Lamb shift; the precession rate is modified by the sum of the modification in $\hat{\mathcal{H}}_{\mathrm{sys}}^{\prime}$ and $\Delta E(i, j)$, where $\Delta E(i, j)$ is the imaginary part of the square brackets in Eq. (7).

\subsection{Writing the Bloch-Redfield equation as a Lindblad equation}

To cast Eq. (5) in the Lindblad form, we rewrite it in terms of a set of orthonormal (trace-class) operators, $\left\{\hat{P}_{i}\right\}$. We use the usual Gram-Schmidt procedure; defining $\hat{P}_{1} \propto \hat{\Gamma}$, and $\hat{P}_{2}$ as proportional to the component of $\hat{\Xi}$ which is orthogonal to $\hat{\Gamma}$. The constants of proportionality are such that both $\hat{P}_{1}$ and $\hat{P}_{2}$ are normalized. Hence

$$
\begin{aligned}
& \hat{P}_{1}=\frac{\hat{\Gamma}}{\sqrt{\operatorname{tr}\left[\hat{\Gamma}^{2}\right]}}, \\
& \hat{P}_{2}=\frac{\hat{\Xi}-\hat{P}_{1} \operatorname{tr}\left[\hat{P}_{1}^{\dagger} \hat{\Xi}\right]}{\sqrt{\operatorname{tr}\left[\hat{\Xi}^{\dagger} \hat{\Xi}\right]-\left|\operatorname{tr}\left[\hat{P}_{1}^{\dagger} \hat{\Xi}\right]\right|^{2}}},
\end{aligned}
$$

so $\hat{P}_{1}$ is Hermitian while in general $\hat{P}_{2}$ is not. As $\hat{P}_{1}, \hat{P}_{2}$ form an orthonormal basis, we have $\hat{\Gamma}=\hat{P}_{1} \operatorname{tr}\left[\hat{P}_{1} \hat{\Gamma}\right]+\hat{P}_{2} \operatorname{tr}\left[\hat{P}_{2} \hat{\Gamma}\right]$ and $\hat{\Xi}=\hat{P}_{1} \operatorname{tr}\left[\hat{P}_{1} \hat{\Xi}\right]+\hat{P}_{2} \operatorname{tr}\left[\hat{P}_{2} \hat{\Xi}\right]$. Then the BlochRedfield equation becomes

$\frac{\mathrm{d}}{\mathrm{d} t} \hat{\rho}(t)=-\mathrm{i}\left[\mathcal{H}_{\mathrm{sys}}^{\prime}, \hat{\rho}(t)\right]-\frac{1}{2} \sum_{i j} h_{i j}\left(\hat{P}_{i}^{\dagger} \hat{P}_{j} \hat{\rho}(t)+\hat{\rho}(t) \hat{P}_{i}^{\dagger} \hat{P}_{j}-2 \hat{P}_{j} \hat{\rho}(t) \hat{P}_{i}^{\dagger}\right)$.

In general, $h_{i j}=\operatorname{tr}\left[\hat{\Gamma}^{\dagger} \hat{P}_{i}\right] \operatorname{tr}\left[\hat{P}_{j}^{\dagger} \hat{\Xi}\right]+\operatorname{tr}\left[\hat{\Xi}^{\dagger} \hat{P}_{i}\right] \operatorname{tr}\left[\hat{P}_{j}^{\dagger} \hat{\Gamma}\right]$. However here $\operatorname{tr}\left[\hat{P}_{2}^{\dagger} \hat{\Gamma}\right]=0$, so $h_{i j}$ is given by the $i j$ th element of the matrix

$$
\mathbf{h}=b_{z}\left(\begin{array}{cc}
1 & 0 \\
0 & 1
\end{array}\right)+\left(\begin{array}{cc}
b_{z} & b_{+} \\
b_{+}^{*} & -b_{z}
\end{array}\right)
$$

where for the compactness of what follows we have defined

$$
\begin{aligned}
& b_{+}=\operatorname{tr}\left[\hat{\Gamma}^{\dagger} \hat{P}_{1}\right] \operatorname{tr}\left[\hat{P}_{2}^{\dagger} \hat{\Xi}\right], \\
& b_{z}=\operatorname{Re}\left(\operatorname{tr}\left[\hat{\Gamma}^{\dagger} \hat{P}_{1}\right] \operatorname{tr}\left[\hat{P}_{1}^{\dagger} \hat{\Xi}\right]\right),
\end{aligned}
$$

we also define $b^{2}=\left|b_{+}\right|^{2}+b_{z}^{2}$. We retain $\dagger \mathrm{s}$ on the symbols to make the structure clear, however $\hat{\Gamma}^{\dagger}=\hat{\Gamma}$ and $\hat{P}_{1}^{\dagger}=\hat{P}_{1} \propto \hat{\Gamma}$. The eigenvalues, $\lambda_{1,2}$, and the $\mathrm{SU}(2)$ rotation, $\mathcal{U}$, 
to the eigenbasis of $\mathbf{h}$, are

$$
\begin{aligned}
\lambda_{1,2} & =b_{z} \pm b \\
\mathcal{U} & =\frac{1}{\sqrt{2}}\left(\begin{array}{cc}
\left(1+b_{z} / b\right)^{1 / 2} & \frac{b_{+}}{\sqrt{b\left(b+b_{z}\right)}} \\
\frac{b_{+}^{*}}{\sqrt{b\left(b+b_{z}\right)}} & -\left(1+b_{z} / b\right)^{1 / 2}
\end{array}\right) .
\end{aligned}
$$

Performing this rotation on Eq. (9), the Bloch-Redfield equation takes the form of the Lindblad equation, Eq. $1 a$, with $\hat{L}_{i}=\sum_{j=1,2} \mathcal{U}_{i j} \hat{P}_{j}$ [45]. However in general $\lambda_{2}$ is negative [14, 15], so this does not satisfy Lindblad's requirement in Eq. (1b).

\section{Perturbative master equation for an extremely short memory time}

We assume here that the memory time, $t_{\mathrm{m}}$, is much shorter than any timescale in $\mathcal{H}_{\text {sys }}$, i.e. $t_{\mathrm{m}} \ll \Delta_{\text {sys }}^{-1}$ where $\Delta_{\text {sys }}$ is the largest energy difference in the system's spectrum. We substitute $\hat{\Gamma}(-\tau)=\hat{\Gamma}-\mathrm{i}\left[\hat{\mathcal{H}}_{\text {sys }}, \hat{\Gamma}\right]_{-} \tau-\frac{1}{2}\left[\hat{\mathcal{H}}_{\text {sys }},\left[\hat{\mathcal{H}}_{\text {sys }}, \hat{\Gamma}\right]_{-}\right]_{-} \tau^{2}+\mathcal{O}\left[\left(\Delta_{\text {sys }} \tau\right)^{3}\right]$ into $\hat{\Xi}$. We expect that $\alpha(\tau)$ is always given by a dimensionless function of $\tau / t_{\mathrm{m}}$ multiplied by $t_{\mathrm{m}}^{-2}$ (given that $\hbar=1$ ). Then $\hat{\Xi}(t)$ (having units of energy) is

$$
\begin{aligned}
\hat{\Xi}(t)= & f_{0}(t) \hat{\Gamma}-\mathrm{i} t_{\mathrm{m}} f_{1}(t)\left[\hat{\mathcal{H}}_{\mathrm{sys}}, \hat{\Gamma}\right]_{-} \\
& -\frac{1}{2} t_{\mathrm{m}}^{2} f_{2}(t)\left[\hat{\mathcal{H}}_{\mathrm{sys}},\left[\hat{\mathcal{H}}_{\mathrm{sys}}, \hat{\Gamma}\right]_{-}\right]_{-}+\mathcal{O}\left[t_{\mathrm{m}}^{-1}\left(\Delta_{\mathrm{sys}} t_{\mathrm{m}}\right)^{3}\right],
\end{aligned}
$$

where $f_{q}(t)=\int_{0}^{t} \mathrm{~d} \tau\left(\tau / t_{\mathrm{m}}\right)^{q} \alpha(\tau)$. For all $q, f_{q}(t)$ goes like $t_{\mathrm{m}}^{-1}$ multiplied by a dimensionless function of $t / t_{\mathrm{m}}$. Thus Eq. (13) is an expansion to second-order in powers of $\Delta_{\text {sys }} t_{\mathrm{m}}$. Writing $f_{q}(t)$ in terms of $S(\omega)$ and $A(\omega)$ we have

$$
f_{q}(t)=\frac{\mathrm{i}}{\left(\mathrm{i} t_{\mathrm{m}}\right)^{q}} \int \frac{\mathrm{d} \omega}{2 \pi}(S(\omega)+A(\omega)) \frac{\mathrm{d}^{q}}{\mathrm{~d} \omega^{q}}\left[\frac{1-\mathrm{e}^{\mathrm{i} \omega t}}{\omega+\mathrm{i} 0^{+}}\right],
$$

where a positive infinitesimal constant, $0^{+}$, ensures the convergence for $t \rightarrow \infty$. Thus

$\hat{\Xi}(t)=\left(f_{0}(t) \sqrt{\operatorname{tr}\left[\hat{\Gamma}^{2}\right]}-\frac{1}{2} t_{\mathrm{m}}^{2} f_{2}(t) K\right) \hat{P}_{1}+t_{\mathrm{m}} f_{1}(t) \sqrt{2 \operatorname{tr}\left[\hat{\Gamma} \hat{\mathcal{H}}_{\mathrm{sys}}\left[\hat{\mathcal{H}}_{\mathrm{sys}}, \hat{\Gamma}\right]_{-}\right]} \hat{P}_{2}$.

The only $f_{2}(t)$-term that we keep is in the prefactor on $\hat{P}_{1}$, for compactness we define $K \equiv \operatorname{tr}\left[\hat{\Gamma}\left[\hat{\mathcal{H}}_{\text {sys }},\left[\hat{\mathcal{H}}_{\text {sys }}, \hat{\Gamma}\right]_{-}\right]_{-}\right] / \sqrt{\operatorname{tr}\left[\hat{\Gamma}^{2}\right]}$. This term gives a $\mathcal{O}\left[t_{\mathrm{m}}^{2}\right]$-term in the final result, while other such $f_{2}(t)$-terms give at worst a $\mathcal{O}\left[t_{\mathrm{m}}^{3}\right]$-term. From Eq. $[11$, we get

$$
\begin{aligned}
& b_{+}=t_{\mathrm{m}} f_{1}(t) \sqrt{2 \operatorname{tr}\left[\hat{\Gamma}^{2}\right] \operatorname{tr}\left[\hat{\Gamma} \hat{\mathcal{H}}_{\mathrm{sys}}\left[\hat{\mathcal{H}}_{\mathrm{sys}}, \hat{\Gamma}\right]_{-}\right]}, \\
& b_{z}=\operatorname{Re}\left[f_{0}(t)\right] \operatorname{tr}\left[\hat{\Gamma}^{2}\right]-\frac{1}{2} t_{\mathrm{m}}^{2} \operatorname{Re}\left[f_{2}(t)\right] K \sqrt{\operatorname{tr}\left[\hat{\Gamma}^{2}\right]} .
\end{aligned}
$$

Since $\operatorname{tr}\left[\hat{\Gamma} \hat{\mathcal{H}}_{\text {sys }}\left[\hat{\mathcal{H}}_{\text {sys }}, \hat{\Gamma}\right]_{-}\right] \lesssim \Delta_{\text {sys }}^{2} \operatorname{tr}\left[\hat{\Gamma}^{2}\right]$, we have $\left|b_{+}\right| \sim\left(\Delta_{\text {sys }} t_{\mathrm{m}}\right) b_{z}$ where $\Delta_{\text {sys }} t_{\mathrm{m}} \ll$ 1. The terms that we dropped only give contributions of order $\left(\Delta_{\mathrm{sys}} t_{\mathrm{m}}\right)^{2} b_{z}$.

To zeroth order in $t_{\mathrm{m}}$ we recover Lindblad's result, Eqs. $(1 a 1 b)$, with only one non-zero coupling constant $\lambda_{1}=2 \operatorname{Re}\left[f_{0}\right] \operatorname{tr}\left[\hat{\Gamma}^{2}\right]>0$, associated with the operator, $\hat{L}_{1}=\left(\operatorname{tr}\left[\hat{\Gamma}^{2}\right]\right)^{-1 / 2} \hat{\Gamma}$. However to first order in $t_{\mathrm{m}}$, we have Eq. $1 a$ with two non-zero coupling constant $\lambda_{1}$ and $\lambda_{2}$; the latter of which is negative (even for infinitesimal $t_{\mathrm{m}}$ ). We use this model to explore the contradiction between Bloch-Redfield and Lindblad. 


\subsection{Environment with a nearly white-noise spectrum}

Here we consider an environment with a nearly white-noise spectrum of excitations (a very wide Lorentzian), at extremely high temperature, $k_{\mathrm{B}} T \gg \omega_{\mathrm{m}}$, so

$$
S(\omega)=S_{0} \frac{\omega_{\mathrm{m}}^{2}}{\omega_{\mathrm{m}}^{2}+\omega^{2}}, \quad A(\omega)=\frac{\omega_{\mathrm{m}} S_{0}}{2 k_{\mathrm{B}} T} \frac{\omega_{\mathrm{m}} \omega}{\omega_{\mathrm{m}}^{2}+\omega^{2}},
$$

where $A(\omega)$ is given by the result below Eq. 6b. . The high-energy cut-off, $\omega_{\mathrm{m}}$, plays the role of the inverse memory time, $t_{\mathrm{m}}^{-1}$, so for nearly white-noise we need it to be much larger than the largest system energy scale, $\Delta_{\text {sys }}$. Then Eq. (14) gives

$f_{0}(t)=\frac{1}{2} S_{0}\left(1+\mathrm{i}\left(2 k_{\mathrm{B}} T t_{\mathrm{m}}\right)^{-1}\right)\left(1-\exp \left[-t / t_{\mathrm{m}}\right]\right)$,

$f_{1}(t)=\frac{1}{2} S_{0}\left(1+\mathrm{i}\left(2 k_{\mathrm{B}} T t_{\mathrm{m}}\right)^{-1}\right)\left(1-\left(1+t / t_{\mathrm{m}}\right) \exp \left[-t / t_{\mathrm{m}}\right]\right)$,

$f_{2}(t)=S_{0}\left(1+\mathrm{i}\left(2 k_{\mathrm{B}} T t_{\mathrm{m}}\right)^{-1}\right)\left(1-\left(1+t / t_{\mathrm{m}}+\left(t / t_{\mathrm{m}}\right)^{2} / 2\right) \exp \left[-t / t_{\mathrm{m}}\right]\right)$,

where we evaluated the $\omega$-integrals using complex analysis (by pushing the contours into the upper-half plane, one finds that the results are due to the pole at $\omega=\mathrm{i} \omega_{\mathrm{m}}$ ). Both $f_{0}(t)$ and $f_{1}(t)$ go exponentially to their long-time limit $\left(t \gg t_{\mathrm{m}}\right)$, with the rate given by the memory time, $t_{\mathrm{m}}$. When $t / t_{\mathrm{m}} \gg 1$ we have $f_{1}(t) / f_{0}(t) \simeq 1$, while when $t / t_{\mathrm{m}} \ll 1$ we have $f_{1}(t) / f_{0}(t) \simeq t / t_{\mathrm{m}}$. For such an environment, the Bloch-Redfield equation is valid for $S_{0} t_{\mathrm{m}} \ll 1$, see Appendix A.

\section{Positivity as a constraint on a two-level system's purity}

To ensure that there is no basis in which the density matrix has negative probabilities (i.e. no possible measurement will return an unphysical probability) it is sufficient and necessary that the density matrix's eigenvalues, $\left\{\Lambda_{k}\right\}$, satisfy $0 \leq \Lambda_{k} \leq 1$ for all $k$. To see this, consider an arbitrary basis which is related to the eigenbasis by the unitary transformation, $\mathcal{U}$. In this basis all probabilities are given by $\hat{\rho}_{i i}=\sum_{k}\left|\hat{\mathcal{U}}_{i k}\right|^{2} \Lambda_{k}$, where the unitarity of $\hat{\mathcal{U}}$ guarantees that $\sum_{k}\left|\hat{\mathcal{U}}_{i k}\right|^{2}=1$. Thus if $0 \leq \Lambda_{k} \leq 1$ for all $k$, then probabilities in this arbitrary basis, satisfy $0 \leq \hat{\rho}_{i i} \leq 1$ for all $i$.

A two-level system is special because the eigenvalues of its density matrix are defined by a single parameter, $s$ (remember that the sum of the eigenvalues must be one). The most general two-by-two density-matrix is of the form $\hat{\rho}=\frac{1}{2}\left(\hat{\sigma}_{0}+\right.$ $\left.s_{x} \hat{\sigma}_{x}+s_{y} \hat{\sigma}_{y}+s_{z} \hat{\sigma}_{z}\right)$, where $\hat{\sigma}_{x, y, z}$ are the Pauli matrices, and $s_{x, y, z}$ are real numbers, when diagonalized it takes the form $\hat{\rho}_{\mathrm{d}}=\frac{1}{2}\left(\hat{\sigma}_{0}+s \hat{\sigma}_{z}\right)$ with the single parameter $s^{2}=s_{x}^{2}+s_{y}^{2}+s_{z}^{2}$. Thus to ensure positivity we require that $-1 \leq s \leq 1$. The purity of $\hat{\rho}$ is $P=\operatorname{tr}\left[\hat{\rho}^{2}\right]=\frac{1}{2}\left(1+s_{x}^{2}+s_{y}^{2}+s_{z}^{2}\right)$, thus ensuring positivity is equivalent to ensuring that $P \leq 1$. This is not the case for systems with more than two levels [46].

Finally it is worth noting that Eq. (1a) leads to

$$
\frac{\mathrm{d} P}{\mathrm{~d} t}=2 \operatorname{tr}\left[\hat{\rho}(t) \frac{\mathrm{d} \hat{\rho}(t)}{\mathrm{d} t}\right]=-2 \sum_{n=0}^{N^{2}-1} \lambda_{n} \operatorname{tr}\left[\hat{L}_{n}^{\dagger}\left[\hat{L}_{n}, \hat{\rho}(t)\right]_{-} \hat{\rho}(t)\right] .
$$

\section{Two-level system with nearly white-noise: proving positivity}

We now consider a two-level system with $\mathcal{H}_{\text {sys }}=-\frac{1}{2} B \hat{\sigma}_{z}$, coupled to an environment via $\hat{\Gamma}=\hat{\sigma}_{x}$. Then Eq. $4 b$ gives $\hat{\Xi}=\int_{0}^{t} \mathrm{~d} \tau \alpha(\tau)\left[\hat{\sigma}_{x} \cos B \tau-\hat{\sigma}_{y} \sin B \tau\right]$, so $\hat{P}_{1}=\hat{\sigma}_{x} / \sqrt{2}$ and $\hat{P}_{2}=\hat{\sigma}_{y} / \sqrt{2}$. For an environment with a short memory time, Eqs. $16 a 16 b$ give 

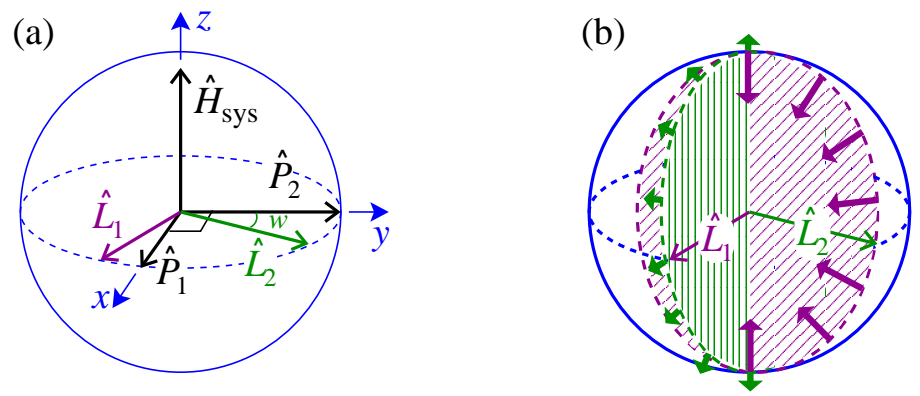

Figure 2. A sketch of the Bloch sphere, for the situation discussed in Section 6 In (a) we show the axes associated with $\hat{\mathcal{H}}_{\text {sys }}, \hat{P}_{1,2}$ and $\hat{L}_{1,2}$. We show $\hat{L}_{1,2}$ for a case where they are Hermitian ( $w$ is real), as only Hermitian operators are associated with axes in the Bloch sphere. In (b) we sketch the effect of the $\hat{L}_{1,2^{-}}$ terms on the evolution of the Bloch vector which represents the density-matrix, $\mathbf{r}_{\text {Bloch }}=\left(2 \operatorname{Re}\left[\rho_{12}\right],-2 \operatorname{Im}\left[\rho_{12}\right], \rho_{11}-\rho_{22}\right)$. The $\hat{L}_{1}$-term reduces the magnitude of the vector in the plane perpendicular to $\hat{L}_{1}$ (diagonal cross-hatching) at a rate given by $\lambda_{1}$. The $\hat{L}_{2}$-term increases the magnitude of the vector in the plane perpendicular to $\hat{L}_{2}$ (vertical cross-hatching) at a rate given by $\left|\lambda_{2}\right| \ll\left|\lambda_{1}\right|$.

$b_{+}=2 B t_{\mathrm{m}} f_{1}(t)$ and $b_{z}=2 \operatorname{Re}\left[f_{0}(t)\right]-\left(B t_{\mathrm{m}}\right)^{2} \operatorname{Re}\left[f_{2}(t)\right]$. Thus to second-order in $B t_{\mathrm{m}}$, Eq. 12 gives

$$
\begin{aligned}
& \lambda_{1}=4 \operatorname{Re}\left[f_{0}(t)\right]+\left(B t_{\mathrm{m}}\right)^{2}\left(\frac{\left|f_{1}(t)\right|^{2}}{\operatorname{Re}\left[f_{0}(t)\right]}-2 \operatorname{Re}\left[f_{2}(t)\right]\right) \\
& \lambda_{2}=-\frac{\left(B t_{\mathrm{m}}\right)^{2}\left|f_{1}(t)\right|^{2}}{\operatorname{Re}\left[f_{0}(t)\right]} .
\end{aligned}
$$

Defining $w=B t_{\mathrm{m}} f_{1}(t) / \operatorname{Re}\left[f_{0}(t)\right]$, the Lindblad operators, $\hat{L}_{1,2}$, are given by

$$
\left(\begin{array}{c}
\hat{L}_{1} \\
\hat{L}_{2}
\end{array}\right)=\mathcal{U}\left(\begin{array}{c}
\hat{P}_{1} \\
\hat{P}_{2}
\end{array}\right)=\frac{1}{\sqrt{2}}\left(\begin{array}{cc}
1-\frac{1}{8}|w|^{2} & w \\
w^{*} & -1+\frac{1}{8}|w|^{2}
\end{array}\right)\left(\begin{array}{c}
\hat{\sigma}_{x} \\
\hat{\sigma}_{y}
\end{array}\right) .
$$

Here we give $\mathfrak{U}$ to first order in $B t_{\mathrm{m}}$, but keep the higher order terms necessary to ensure $\mathcal{U}^{\dagger} \mathcal{U}=1$. Note that $\hat{L}_{1}$ and $\hat{L}_{2}$ are not Hermitian unless $f_{1}(t)$ is real.

We take the $T \rightarrow \infty$ limit of the nearly white-noise in Section 4.1 so $\operatorname{Im}\left[f_{1}(t)\right]=0$, then $w$ is real and gives the angle marked in Fig. $2 a$. Defining the $x^{\prime}, y^{\prime}$-axes such that $\hat{L}_{1}=\hat{\sigma}_{x^{\prime}} / \sqrt{2}$ and $\hat{L}_{2}=\hat{\sigma}_{y^{\prime}} / \sqrt{2}$, the Bloch-Redfield equation reduces to

$\frac{\mathrm{d}}{\mathrm{d} t} \hat{\rho}(t)=-\mathrm{i}\left[\hat{\mathcal{H}}_{\mathrm{sys}}^{\prime}, \hat{\rho}(t)\right]-2 \lambda_{1}\left(\hat{\rho}(t)-\hat{\sigma}_{x^{\prime}} \hat{\rho}(t) \hat{\sigma}_{x^{\prime}}\right)-2 \lambda_{2}\left(\hat{\rho}(t)-\hat{\sigma}_{y^{\prime}} \hat{\rho}(t) \hat{\sigma}_{y^{\prime}}\right)$.

The coupling constants, $\lambda_{1}, \lambda_{2}$, are given by Eqs. 18a $18 b 20$ with $T \rightarrow \infty$, so

$$
\begin{aligned}
& \lambda_{1}=2 S_{0}\left(1-\mathrm{e}^{-t / t_{\mathrm{m}}}\right)+\mathcal{O}\left[\left(B t_{\mathrm{m}}\right)^{2}\right], \\
& \lambda_{2}=-\left(B t_{\mathrm{m}}\right)^{2} S_{0} \frac{\left(1-\left(1+t / t_{\mathrm{m}}\right) \mathrm{e}^{-t / t_{\mathrm{m}}}\right)^{2}}{2\left(1-\mathrm{e}^{-t / t_{\mathrm{m}}}\right)} .
\end{aligned}
$$

Substituting these results into Eq. (19), and writing $-\lambda_{2}$ as $+\left|\lambda_{2}\right|$ to emphasis that it tends to increase the purity, we get

$$
\frac{\mathrm{d} P}{\mathrm{~d} t}=-\lambda_{1} \operatorname{tr}\left[\hat{\rho}^{2}(t)-\left(\hat{\sigma}_{x^{\prime}} \hat{\rho}(t)\right)^{2}\right]+\left|\lambda_{2}\right| \operatorname{tr}\left[\hat{\rho}^{2}(t)-\left(\hat{\sigma}_{y^{\prime}} \hat{\rho}(t)\right)^{2}\right] .
$$




\subsection{Positivity at short times (times of order the memory time)}

For times, $t$, much less than $S_{0}^{-1}$ we can get the purity to first order in $S_{0}$, by integrating Eq. (24) with $\hat{\rho}(t)$ replaced by its value to zeroth order in $S_{0}$

$\hat{\rho}^{(0)}(t)=\frac{1}{2}\left[1+\left(s_{x} \cos B t+s_{y} \sin B t\right) \hat{\sigma}_{x^{\prime}}+\left(s_{y} \cos B t-s_{x} \sin B t\right) \hat{\sigma}_{y^{\prime}}+s_{z} \hat{\sigma}_{z}\right]$,

where the constants $\left(s_{x}, s_{y}, s_{z}\right)$ define $\hat{\rho}(t=0)$. Note that we have used the fact that to zeroth order in $S_{0}$ we have $\hat{\mathcal{H}}_{\text {sys }}^{\prime}=\hat{\mathcal{H}}_{\text {sys }}=-\frac{1}{2} B \hat{\sigma}_{z}$. Then Eq. 24 becomes

$$
\begin{gathered}
\frac{\mathrm{d} P(t)}{\mathrm{d} t}=-\lambda_{1}\left[\left(s_{y} \cos B t-s_{x} \sin B t\right)^{2}+s_{z}^{2}\right]+\left|\lambda_{2}\right|\left[\left(s_{x} \cos B t+s_{y} \sin B t\right)^{2}+s_{z}^{2}\right] \\
+\mathcal{O}\left[S_{0}^{2} t\right] .
\end{gathered}
$$

As $t_{\mathrm{m}} \ll B^{-1}$, we can restrict ourselves to times $t \ll B^{-1}, S_{0}^{-1}$ (and hence expand in powers of $B t$ and $S_{0} t$ ), and still study the dynamics up to times $\gg t_{\mathrm{m}}$. The problematic coupling constant, $\lambda_{2}$, is $\mathcal{O}\left[B^{2}\right]$, so we must expand the right-hand-side of Eq. 26 to $\mathcal{O}\left[B^{2}\right]$, to see the effect of $\lambda_{2}$ on the dynamics. After this expansion in $B$, we expand the purity about $P(0)=1$. So $P(t)=1+\int_{0}^{t} \mathrm{~d} t^{\prime}\left(\mathrm{d} P\left(t^{\prime}\right) / \mathrm{d} t^{\prime}\right)$ gives

$P(t)=1-2 S_{0} t_{\mathrm{m}}\left[s_{z}^{2} I_{z}+s_{y}^{2} I_{y}-2 s_{x} s_{y} B t_{\mathrm{m}} I_{x y}+s_{x}^{2}\left(B t_{\mathrm{m}}\right)^{2} I_{x}\right]+\mathcal{O}\left[S_{0}^{2} t, S_{0} B^{3} t^{3}\right]$

where $I_{z}, I_{y}, I_{x y}$ and $I_{x}$ are the following functions of $t / t_{\mathrm{m}}$,

$$
\begin{aligned}
I_{z}\left(t / t_{\mathrm{m}}\right) & \int_{0}^{t} \mathrm{~d} t^{\prime} \frac{\lambda_{1}\left(t^{\prime}\right)+\lambda_{2}\left(t^{\prime}\right)}{2 S_{0} t_{\mathrm{m}}} & \simeq \int_{0}^{t / t_{\mathrm{m}}} \mathrm{d} \nu\left(1-\mathrm{e}^{-\nu}\right), \\
I_{y}\left(t / t_{\mathrm{m}}\right) \equiv & \int_{0}^{t} \mathrm{~d} t^{\prime} \frac{\left(1-\left(B t^{\prime}\right)^{2}\right) \lambda_{1}\left(t^{\prime}\right)}{2 S_{0} t_{\mathrm{m}}} & \simeq \int_{0}^{t / t_{\mathrm{m}}} \mathrm{d} \nu\left(1-\mathrm{e}^{-\nu}\right), \\
I_{x y}\left(t / t_{\mathrm{m}}\right) \equiv & \int_{0}^{t} \mathrm{~d} t^{\prime} \frac{t^{\prime} \lambda_{1}\left(t^{\prime}\right)}{2 S_{0} t_{\mathrm{m}}^{2}} & \simeq \int_{0}^{t / t_{\mathrm{m}}} \mathrm{d} \nu \nu\left(1-\mathrm{e}^{-\nu}\right), \\
I_{x}\left(t / t_{\mathrm{m}}\right) \equiv & \int_{0}^{t} \mathrm{~d} t^{\prime} \frac{\left(B t^{\prime}\right)^{2} \lambda_{1}\left(t^{\prime}\right)+\lambda_{2}\left(t^{\prime}\right)}{2 S_{0} B^{2} t_{\mathrm{m}}^{3}} & \simeq \int_{0}^{t / t_{\mathrm{m}}} \mathrm{d} \nu\left[\nu^{2}\left(1-\mathrm{e}^{-\nu}\right)-\frac{\left[1-(1+\nu) \mathrm{e}^{-\nu}\right]^{2}}{4\left(1-\mathrm{e}^{-\nu}\right)}\right],
\end{aligned}
$$

where $\nu=t^{\prime} / t_{\mathrm{m}}$. The " $\simeq$ " indicates that we keep only the leading order in $\left(B t_{\mathrm{m}}\right)$ in each term, this will be sufficient for our purposes.

To show that $P(t)$ does not exceed one (in the range of $t$ for which Eq. (27) is valid), we show that the square-bracket in Eq. (27) is never negative. Writing the square-bracket as $\left[I_{z} s_{z}^{2}+I_{y}\left(s_{y}-s_{x}\left(B t_{\mathrm{m}}\right) I_{x y} / I_{y}\right)^{2}+s_{x}^{2}\left(B t_{\mathrm{m}}\right)^{2}\left(I_{x}-I_{x y}^{2} / I_{y}\right)\right]$, we see that there are three terms; the first two are always positive (but will be small for spins starting close to the $x^{\prime}$-axis, i.e. $\left.s_{y}, s_{z} \ll 1\right)$, the third term is positive if $I_{x}>I_{x y}^{2} / I_{y}$. Thus we must show that $I_{y} I_{x} / I_{x y}^{2} \geq 1$. For $t \ll t_{\mathrm{m}}$,

$$
I_{y} \rightarrow \frac{1}{2}\left(t / t_{\mathrm{m}}\right)^{2} \quad I_{x y} \rightarrow \frac{1}{3}\left(t / t_{\mathrm{m}}\right)^{3} \quad I_{x} \rightarrow \frac{15}{64}\left(t / t_{\mathrm{m}}\right)^{4},
$$

and for $t \gg t_{\mathrm{m}}$,

$$
I_{y} \rightarrow t / t_{\mathrm{m}} \quad I_{x y} \rightarrow \frac{1}{2}\left(t / t_{\mathrm{m}}\right)^{2} \quad I_{x} \rightarrow \frac{1}{3}\left(t / t_{\mathrm{m}}\right)^{3} .
$$

Thus for $t \ll t_{\mathrm{m}}$ we have $I_{y} I_{x} / I_{x y}^{2} \rightarrow 135 / 128$, while for $t \gg t_{\mathrm{m}}$ we have $I_{y} I_{x} / I_{x y}^{2} \rightarrow$ $4 / 3$. For finite $t$ we see that $I_{y} I_{x} / I_{x y}^{2}$ is a monotonic function of $t$ which goes from 


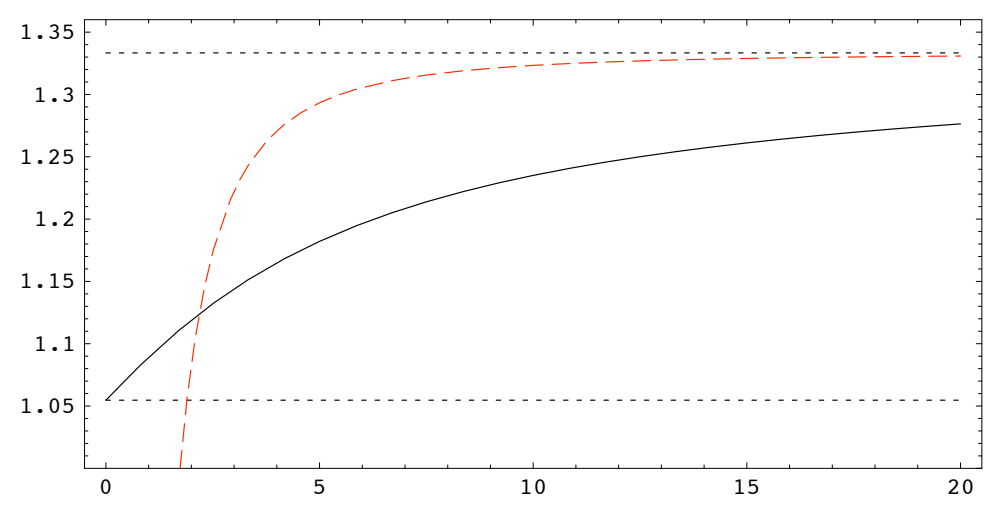

Figure 3. Plots of $I_{y} I_{x} / I_{x y}^{2}$ (solid curve) and $I_{y}^{(\infty)} I_{x}^{(\infty)} /\left(I_{x y}^{(\infty)}\right)^{2}$ (dashed curve) as functions of $t / t_{\mathrm{m}}$. The two horizontal lines are the two extrema of $I_{y} I_{x} / I_{x y}^{2}$; its small $t$ limit of $135 / 128$ and its large $t$ limit of $4 / 3$. The crucial point is that $I_{y} I_{x} / I_{x y}^{2}>1$ for all $t \geq 0$. This is not the case for $I_{y}^{(\infty)} I_{x}^{(\infty)} /\left(I_{x y}^{(\infty)}\right)^{2}$, which one would get if one mistakenly assumed time-independent coupling constants $\lambda_{1}(\infty), \lambda_{2}(\infty)$; this is less than one for all $t / t_{\mathrm{m}}<\sqrt{3}$ and goes to $-\infty$ at $t=0$.

$135 / 128$ to $4 / 3$ (see Fig. 3), thus it is always greater than one. This means that $P(t) \leq 1$ for all times much less than $S_{0}^{-1}$ (including times greater than $t_{\mathrm{m}}$ ).

For $t_{\mathrm{m}} \ll t \ll B^{-1}, S_{0}^{-1}$, the purity $P(t)=1-2 S_{0} t\left[s_{z}^{2}+\left(s_{y}-\frac{1}{2} s_{x} B t\right)^{2}+\right.$ $\left.\frac{1}{12}\left(s_{x} B t\right)^{2}\right]$. By checking all pure initial system states (state with $s_{x}^{2}+s_{y}^{2}+s_{z}^{2}=1$ ) we see that this is maximal for $s_{x}= \pm\left[1+(B t)^{2} / 8\right]+\mathcal{O}\left[B^{3} t^{3}\right], s_{y}= \pm \frac{1}{2} B t$ and $s_{z}=0$ (where $s_{x}$ and $s_{y}$ have the same sign) [47]. Hence

$$
P\left(t_{\mathrm{m}} \ll t \ll B^{-1}, S_{0}^{-1}\right) \leq 1-\frac{1}{6} S_{0} B^{2} t^{3} .
$$

This upper-bound on the purity will be crucial for our proof (in section 6.2 that the purity does not exceed one at longer times.

If we had made the usual assumption that one can replace $\lambda_{1}(t)$ and $\lambda_{2}(t)$ with $\lambda_{1}(t=\infty)$ and $\lambda_{2}(t=\infty)$ for all $t$, then we would have Eqs. $\left.28 a \mid 28 d\right)$ with $I_{y}, I_{x y}, I_{x}$ replaced by $I_{y}^{(\infty)}=\int_{0}^{t / t_{\mathrm{m}}} \mathrm{d} \nu, I_{x y}^{(\infty)}=\int_{0}^{t / t_{\mathrm{m}}} \mathrm{d} \nu \nu$, and $I_{x}^{(\infty)}=\int_{0}^{t / t_{\mathrm{m}}} \mathrm{d} \nu\left[\nu^{2}-1 / 4\right]$. We plot $I_{y}^{(\infty)} I_{x}^{(\infty)} /\left(I_{x y}^{(\infty)}\right)^{2}$ in Fig. 3. and see that it goes to $-\infty$ as $t / t_{\mathrm{m}} \rightarrow 0$. Thus such a mistaken assumption would have led us to conclude (as other have) that $P$ can become bigger than one (at times $\lesssim t_{\mathrm{m}}$ ). The mistake is most clearly illustrated by looking at Eq. 27. with $s_{x}=1$ and $s_{y}=s_{z}=0$, then using $I_{x}^{(\infty)}$ in place of $I_{x}$ would lead one to think that for $t \ll t_{\mathrm{m}}$, the purity would be $1+\frac{1}{2} S_{0} t$ when the correct expression shows it is $1-\frac{2}{3} S_{0} t_{\mathrm{m}}\left(t / t_{\mathrm{m}}\right)^{3}$. Thus it is only by keeping the time-dependence of the coupling constants, that we can show that the purity cannot exceed one for all times $\ll S_{0}^{-1}$ (including times greater than $t_{\mathrm{m}}$ ).

\subsection{Positivity at long times (times of order and greater than $S_{0}^{-1}$ )}

We now turn to the evolution of the purity at all times much greater than $t_{\mathrm{m}}$ (the long-time regime in Fig. 1). For times of order and greater than $S_{0}^{-1}$ we need the full Bloch-Redfield equation, Eq. 22), not just the short time expansion of it. Since $t \gg t_{\mathrm{m}}$, the coupling constants have saturated at their long time limits; $\lambda_{1}=2 S_{0}$ and 
$\lambda_{2}=-2 S_{0}\left(B t_{\mathrm{m}} / 2\right)^{2}$. Then Eq. 24 reduces to

$$
\frac{\mathrm{d} P(t)}{\mathrm{d} t}=-2 S_{0}\left[\left(1-\left(B t_{\mathrm{m}} / 2\right)^{2}\right) s_{z}^{2}(t)+s_{y}^{2}(t)-\left(B t_{\mathrm{m}} / 2\right)^{2} s_{x}^{2}(t)\right] .
$$

Since $B t_{\mathrm{m}} \ll 1$, we can see that $P\left(t \gg t_{\mathrm{m}}\right)$ decays for nearly all $s_{x, y, z}(t)$. However the purity may grow if $s_{y}(t) \sim s_{z}(t) \sim \mathcal{O}\left[\left(B t_{\mathrm{m}}\right)^{2}\right]$; then the purity might exceed one (particularly if $s_{x}$ is close to one).

To see if the purity can exceed one, we expand the evolution about the time $t_{0}$, where we choose $t_{0}$ such that $s_{y}\left(t_{0}\right)=0$. We then perform the same expansion about $t=t_{0}$ here as we performed about $t=0$ in Section 6.1. Hence on the right-hand-side of Eq. (32) we make the substitution $s_{x}\left(t_{0}+\tau\right)=s_{x}^{\prime} \cos B \tau, s_{y}\left(t_{0}+\tau\right)=s_{x}^{\prime} \sin B \tau$, $s_{z}\left(t_{0}+\tau\right)=s_{z}^{\prime}$, where we define $s_{x}^{\prime}=s_{x}\left(t_{0}\right)$ and $s_{z}^{\prime}=s_{z}\left(t_{0}\right)$ (remember that $t_{0}$ is chosen such that $s_{y}\left(t_{0}\right)=0$ ). This substitution is good for all $\tau \ll S_{0}^{-1}$, After the substitution we expand the right-hand-side of Eq. (32) up to second order in $B \tau$. Thus for $\tau \ll B^{-1}, S_{0}^{-1}$,

$$
\left.\frac{\mathrm{d} P(t)}{\mathrm{d} t}\right|_{t=t_{0}+\tau}=-2 S_{0}\left[\left(1-\left(B t_{\mathrm{m}} / 2\right)^{2}\right){s_{z}^{\prime}}^{2}+\left(s_{x}^{\prime} B \tau\right)^{2}-\left(s_{x}^{\prime} B t_{\mathrm{m}} / 2\right)^{2}\right] .
$$

From this we see that the purity can only increase during a time-window where $|\tau|<\left[\left(t_{\mathrm{m}} / 2\right)^{2}-\left(s_{z}^{\prime} / s_{x}^{\prime} B\right)^{2}\right]^{1 / 2}$ (neglecting a term that is higher order in $\left.B t_{\mathrm{m}}\right)$. The maximum possible time for this growth is $t_{\mathrm{m}}$ (i.e. when $s_{z}^{\prime}=0, P$ grows during the time-window from $\tau=-\frac{1}{2} t_{\mathrm{m}}$ to $\left.\tau=\frac{1}{2} t_{\mathrm{m}}\right)$. Thus the assumption that $\tau \ll B^{-1}, S_{0}^{-1}$ is fulfilled for all $\tau$ at which the purity is growing.

At this point it is sufficient to make a gross over-estimate of the amount by which the purity can grow. If we assumed that the purity grows during the entire timewindow $-\frac{1}{2} t_{\mathrm{m}} \leq \tau \leq \frac{1}{2} t_{\mathrm{m}}$ at the maximal possible rate (i.e. the rate at $\tau=0$ when $s_{z}^{\prime}=0$ and $s_{x}=1$ ), then during this time-window it would grow by $\frac{1}{2} S_{0} B^{2} t_{\mathrm{m}}^{3}$. If we define $\Delta P$ as the true increase of the purity in the time-window where it grows, the over-estimate enables us to put the following upper-bound;

$$
\Delta P<\frac{1}{2} S_{0} B^{2} t_{\mathrm{m}}^{3}
$$

Comparing this with the upper-bound on the purity in Eq. (31) with $t \gg t_{\mathrm{m}}$ (but $t \ll S_{0}^{-1}$ ), we see that increasing the purity by $\Delta P$ cannot cause it to exceed one. The short- and long-time regimes overlap (see Fig. 1), so by showing that $P \leq 1$ in both regimes we have shown positivity for all $t>0$.

\section{Conclusions}

The Bloch-Redfield master equation for an arbitrary system can be written in the form of a Lindblad master equation, Eq. $11 a$. Only by setting the memory time equal to zero (strictly Markovian evolution) do we recover Lindblad's result with coupling constants, $\left\{\lambda_{n}\right\}$, which are time-independent and positive, Eq. $1 b$.

For finite memory times, the Bloch-Redfield master equation can still be cast in the form of Eq. $1 a$, but its do not satisfy Eq. $11 b$. However, the parameters are time-dependent which means that the semigroup property is absent, and so Lindblad's requirements are inapplicable. We show analytically for a particular model (a two-level system coupled to a high-temperature environment with a memory time much less than system timescales) that the master equation preserves positivity if and only if we keep the time-dependence of the parameters. Further, it turns out that positivity and complete positivity are equivalent for this particular model [6]. 
It is remarkable that our result only coincides with Lindblad's for strictly zero memory time, $t_{\mathrm{m}}=0$. If we take the limit $t_{\mathrm{m}} \rightarrow 0$, we find that one coupling constant tends to zero from below. Further, we argue (see the appendix) that the Bloch-Redfield equations become exact in this limit. Thus even for infinitesimal $t_{\mathrm{m}}$, one coupling constant is negative. Positivity (and hence complete positivity) is nonethe-less preserved by the time-dependence of the coupling constants at times of order the infinitesimal time $t_{\mathrm{m}}$.

We wonder if an analysis of the time-dependent parameters of an arbitrary BlochRedfield master equation would show positivity, or even complete positivity. If this could be proven, one could argue that the Bloch-Redfield master equation contains both the Lindblad equation and finite memory-time corrections to it.

\section{Acknowledgments}

This work was stimulated by conversations with J. Siewert, Y. Gefen and S. Stenholm, at the "Workshop on entanglement, decoherence and geometric phases in complex systems", Abdus Salam ICTP, 2004. I am extremely grateful to M. Hall for pointing out a serious mistake in the first draft of this manuscript, and for enlightening discussions. My thanks also go to A. Shnirman, M. Clusel and D. O'Dell for useful discussions. The Swiss NSF financed early stages of this work, part of which was carried out at the Aspen Centre for Physics.

\section{Appendix A. Deriving Bloch-Redfield from a Dyson equation}

For completeness, we sketch the derivation of the Bloch-Redfield master equation [9, 10, using a common "modern" approach [41 based on a real-time Dyson equation [13. The derivation is none-the-less equivalent to Refs. 9, 10]. At $t=0$ the system and environment are in a factorized state (e.g. a perfect projective measurement is made on the system at $t=0)$. The propagator of the system's reduced density matrix is $\mathbb{K}_{i^{\prime} j^{\prime} ; i j}(t ; 0)=\operatorname{tr}_{\text {env }}\left[\left\langle i^{\prime}\left|\mathrm{e}^{-\mathrm{i} \hat{\mathcal{H}}_{\text {univ }} t}\right| i\right\rangle \hat{\rho}_{\text {env }}\left\langle j\left|\mathrm{e}^{\mathrm{i} \hat{\mathcal{H}}_{\text {univ }} t}\right| j^{\prime}\right\rangle\right]$, with Eq. 22 giving the system's reduced density-matrix at time $t$. The Dyson equation for $\mathbb{K}\left(t ; t_{0}\right)$ (treating the system-environment interaction as a perturbation, which we keep to all orders) is

$\mathbb{K}(t ; 0)=\mathbb{K}^{\mathrm{sys}}(t ; 0)+\int_{0}^{t} \mathrm{~d} t_{2} \int_{0}^{t_{2}} \mathrm{~d} t_{1} \mathbb{K}^{\mathrm{sys}}\left(t ; t_{2}\right) \boldsymbol{\Sigma}\left(t_{2} ; t_{1}\right) \mathbb{K}^{\mathrm{sys}}\left(t_{1} ; 0\right)$,

where $\mathbb{K}\left(t ; t^{\prime}\right)$ is the propagator including all interactions; $\mathbb{K}^{\text {sys }}\left(t ; t^{\prime}\right)$ is the bare system propagator (propagating it only under the Hamiltonian $\hat{\mathcal{H}}_{\text {sys }}$ ). Since there are no interactions after $t=0$ in the first term and after time $t_{2}$ in the second term above, we can trace out the environment at these times. Finally $\boldsymbol{\Sigma}\left(t_{2} ; t_{1}\right)$ an irreducible block of the propagator (with the same tensor structure as $\mathbb{K}(t ; 0)$ ), it is the smallest block for which the system has interacted with one or more environment excitations.

Taking the time-derivative of Eq. A.1), and noting that $(\mathrm{d} / \mathrm{d} t) \int_{0}^{t} \mathrm{~d} t_{2} \mathbb{K}^{\text {sys }}\left(t ; t_{2}\right) \mathbb{F}\left(t_{2}\right)$ $=\mathbb{F}(t)+\int_{0}^{t} \mathrm{~d} t_{2} \mathbb{E}^{\text {sys }}(t) \mathbb{K}^{\text {sys }}\left(t ; t_{2}\right) \mathbb{F}\left(t_{2}\right)$ for any $\mathbb{F}\left(t_{2}\right)$, we get the master equation

$$
\frac{\mathrm{d}}{\mathrm{d} t} \mathbb{K}(t ; 0)=-\mathrm{i} \mathbb{E}^{\mathrm{sys}}(t) \mathbb{K}(t ; 0)+\int_{0}^{t} \mathrm{~d} t_{1} \boldsymbol{\Sigma}\left(t ; t_{1}\right) \mathbb{K}\left(t_{1} ; 0\right) .
$$

We have defined $\mathbb{E}_{i^{\prime} j^{\prime} ; i j}^{\text {ss }}=\left\langle i^{\prime}\left|\mathcal{H}_{\text {sys }}\right| i\right\rangle\left\langle j\left|\mathcal{H}_{\text {sys }}\right| j^{\prime}\right\rangle$, then $(\mathrm{d} / \mathrm{d} t) \mathbb{K}^{\text {sys }}(t ; 0)=$ $-\mathrm{i} \mathbb{E}^{\text {sys }}(t) \mathbb{K}^{\text {sys }}(t ; 0)$. To clearly see the non-Markovian nature of Eq. A.2 we can 
(a)

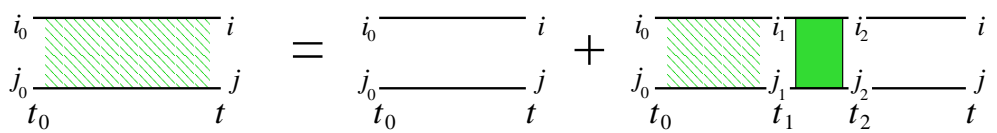

(b)

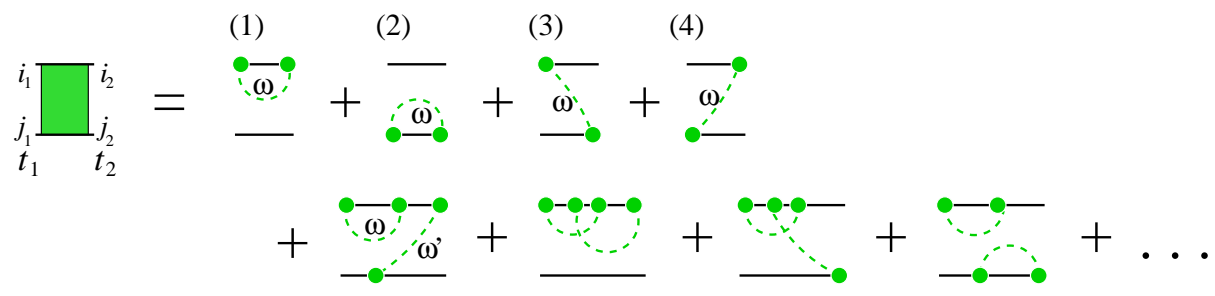

Figure A1. (a) Real time Dyson equation for an arbitrary system. The pair of lines with cross-hatching between them are the full propagator, $\mathbb{K}\left(t^{\prime \prime} ; t^{\prime}\right)$; the lines without cross-hatching are the bare system propagator, $\mathbb{K}^{\text {sys }}\left(t^{\prime \prime} ; t^{\prime}\right)$; and the lines with solid colour between them are the irreducible block, $\boldsymbol{\Sigma}\left(t^{\prime \prime}, t^{\prime}\right)$. Internal indices are summed over and internal times are integrated over as in Eq. A.1. This drawing of the propagators emphasizes that only $\mathbb{K}_{i^{\prime \prime}, j^{\prime \prime} ; i^{\prime} j^{\prime}}^{\mathrm{sys}}\left(t^{\prime \prime} ; t^{\prime}\right)$ can be written in the form $A_{i^{\prime \prime} ; i^{\prime}} \times B_{j^{\prime \prime} ; j^{\prime}}$. (b) Some lower-order diagrams for the irreducible block, $\boldsymbol{\Sigma}\left(t^{\prime \prime}, t^{\prime}\right)$, in all cases we integrate $\omega, \omega^{\prime}$, etc, over the spectrum of excitations. The second-order diagrams are labelled (1) to (4).

substitute it into Eq. 22 which gives $(\mathrm{d} / \mathrm{d} t) \hat{\rho}(t)=-\mathrm{i}\left[\hat{\mathcal{H}}_{\text {sys }}(t), \hat{\rho}(t)\right]+\int_{0}^{t} \mathrm{~d} t_{1} \boldsymbol{\Sigma}\left(t ; t_{1}\right) \hat{\rho}\left(t_{1}\right)$. This master equation is exact, our only assumption was that the system and environment were in a factorized state at time $t=0$. It is formally equivalent to the Nakajima-Zwanzig equation [11, 12. However it is of little practical use (giving no great advantage over standard perturbation theory) unless the irreducible block, $\boldsymbol{\Sigma}\left(t_{2} ; t_{1}\right)$ is reasonably local in time, i.e. decays on a scale $t_{2}-t_{1} \ll t$. Without approximation we can use $\hat{\rho}(t)=\mathbb{K}\left(t ; t_{1}\right) \hat{\rho}\left(t_{1}\right)$ to write this master equation as

$$
\frac{\mathrm{d}}{\mathrm{d} t} \hat{\rho}(t)=-\mathrm{i}\left[\hat{\mathcal{H}}_{\mathrm{sys}}(t), \hat{\rho}(t)\right]+\int_{0}^{t} \mathrm{~d} t_{1} \boldsymbol{\Sigma}\left(t ; t_{1}\right) \mathbb{K}^{-1}\left(t ; t_{1}\right) \hat{\rho}(t) .
$$

This might "look" Markovian, but the non-Markovian nature is in the new term $\mathbb{K}^{-1}\left(t ; t_{1}\right)$. Approximations of Eq. A.3 will give a Bloch-Redfield master equation.

\section{Appendix A.1. The Bloch-Redfield equation from a Born approximation}

Here we get the Bloch-Redfield master equation by making a Born approximation of the irreducible block $\boldsymbol{\Sigma}\left(t^{\prime \prime}, t^{\prime}\right)$ in Eq. A.3). It involves neglecting all contributions to $\boldsymbol{\Sigma}\left(t^{\prime \prime}, t^{\prime}\right)$ beyond second-order. Our derivation involves two assumptions which justify the Born approximation (other derivations may be possible).

Our first assumption is that the environment is large enough to have a continuous energy-spectrum of excitations (although it does not matter if this spectrum is discrete on scales $\left.\ll t^{-1}\right)$. So for finite relaxation/decoherence rates, we assume the coupling to each environment excitation is small enough to be treated only up to second order. Thus each excitation evolves only under $\hat{\mathcal{H}}_{\text {env }}$ up to the time of its (first or second order) interaction with the system. It then never interacts with the system again, so we trace it out immediately after the (first or second order) interaction.

Our second assumption is that the environment's initial density-matrix obeys $\left[\hat{\mathcal{H}}_{\text {env }}, \hat{\rho}_{\text {env }}\right]=0$, as would be the case for either an eigenstate or any classical mixture of 
eigenstates of $\hat{\mathcal{H}}_{\text {env }}$ (such as a thermal state). Combining this with our first assumption means that we can treat $\hat{\rho}_{\text {env }}$ as time-independent. Then without loss of generality we can make $\operatorname{tr}$ env $\left[\hat{x} \hat{\rho}_{\text {env }}\right]=0$, by moving any constant off-set into the definition of $\hat{\mathcal{H}}_{\text {sys }}$. This removes the first order contributions from the irreducible block, $\boldsymbol{\Sigma}\left(t^{\prime \prime}, t^{\prime}\right)$. Thus $\boldsymbol{\Sigma}\left(t^{\prime \prime}, t^{\prime}\right)$ becomes the sum of second-order (and higher-order) terms sketched in Fig. A1p. The dotted lines indicate that a given environment excitation (with energy $\omega)$ has been created by the system-environment interaction.

Treating the integral in Eq. A.3 to lowest (second) order in $\hat{x}$, means making a Born approximation on $\boldsymbol{\Sigma}\left(t^{\prime \prime}, t^{\prime}\right)$ and treating $\mathbb{K}^{-1}\left(t ; t_{1}\right)$ to zeroth order in $\hat{x}$ [48]. Hence defining $\tau=t-t_{1}$ and $\mathbb{S}(\tau)=\boldsymbol{\Sigma}^{\text {Born }}(t ; t-\tau)\left[\mathbb{K}^{\text {sys }}(t ; t-\tau)\right]^{-1}$, we have

$$
\frac{\mathrm{d}}{\mathrm{d} t} \hat{\rho}(t)=-\mathrm{i}\left[\hat{\mathcal{H}}_{\mathrm{sys}}(t), \hat{\rho}(t)\right]+\int_{0}^{t} \mathrm{~d} \tau \mathbb{S}(\tau) \hat{\rho}(t) .
$$

The four contributions to $\boldsymbol{\Sigma}^{\text {Born }}(t ; t-\tau)$, labelled (1-4) in Fig. A1b, give

$$
\begin{aligned}
& \mathbb{S}_{i^{\prime} j^{\prime} ; i j}^{(1)}(\tau)=\operatorname{tr}_{\text {env }}\left[\left\langle i^{\prime}\left|\hat{\Gamma} \hat{x} \mathrm{e}^{-\mathrm{i} \hat{\mathcal{H}}_{0} \tau} \hat{\Gamma} \hat{x} \mathrm{e}^{\mathrm{i} \hat{\mathcal{H}}_{0} \tau}\right| i\right\rangle \rho_{\text {env }}\left\langle j \mid j^{\prime}\right\rangle\right], \\
& \mathbb{S}_{i^{\prime} j^{\prime} ; i j}^{(2)}(\tau)=\operatorname{tr}_{\text {env }}\left[\left\langle i^{\prime} \mid i\right\rangle \rho_{\mathrm{env}}\left\langle j\left|\mathrm{e}^{-\mathrm{i} \hat{\mathcal{H}}_{0} \tau} \hat{\Gamma} \hat{x} \mathrm{e}^{\mathrm{i} \hat{\mathcal{H}}_{0} \tau} \hat{\Gamma} \hat{x}\right| j^{\prime}\right\rangle\right], \\
& \mathbb{S}_{i^{\prime} j^{\prime} ; i j}^{(3)}(\tau)=\operatorname{tr}_{\text {env }}\left[\left\langle i^{\prime}\left|\mathrm{e}^{-\mathrm{i} \hat{\mathcal{H}}_{0} \tau} \hat{\Gamma} \hat{x} \mathrm{e}^{\mathrm{i} \hat{\mathcal{H}}_{0} \tau}\right| i\right\rangle \rho_{\text {env }}\left\langle j|\hat{\Gamma} \hat{x}| j^{\prime}\right\rangle\right], \\
& \mathbb{S}_{i^{\prime} j^{\prime} ; i j}^{(4)}(\tau)=\operatorname{tr}_{\text {env }}\left[\left\langle i^{\prime}|\hat{\Gamma} \hat{x}| i\right\rangle \rho_{\mathrm{env}}\left\langle j\left|\mathrm{e}^{\mathrm{i} \hat{\mathcal{H}}{ }_{0} \tau} \hat{\Gamma} \hat{x} \mathrm{e}^{-\mathrm{i} \hat{\mathcal{H}}_{0} \tau}\right| j^{\prime}\right\rangle\right],
\end{aligned}
$$

where we define $\hat{\mathcal{H}}_{0}=\hat{\mathcal{H}}_{\text {sys }}+\hat{\mathcal{H}}_{\text {env }}$. We re-write all these contributions in terms of operators acting to the left and right of the density-matrix, $\hat{\rho}(t)$. Those interaction on the upper line are to the left of $\hat{\rho}(t)$, while those on the lower line are to the right. Thus summing these four terms we get $\mathbb{S}(\tau) \hat{\rho}(t)=\operatorname{tr}_{\text {env }}[\hat{\Gamma}(0) \hat{x}(0) \hat{\Gamma}(-\tau) \hat{x}(-\tau)[\hat{\rho}(t) \otimes$ $\left.\rho_{\text {env }}\right]+\left[\hat{\rho}(t) \otimes \rho_{\text {env }}\right] \hat{\Gamma}(-\tau) \hat{x}(-\tau) \hat{\Gamma} \hat{x}+\hat{\Gamma}(-\tau) \hat{x}(-\tau)\left[\hat{\rho}(t) \otimes \rho_{\text {env }}\right] \hat{\Gamma}(0) \hat{x}(0)+\hat{\Gamma}(0) \hat{x}(0)[\hat{\rho}(t) \otimes$ $\left.\left.\rho_{\text {env }}\right] \hat{\Gamma}(-\tau) \hat{x}(-\tau)\right]$, where the operators are in the interaction picture, so $\Gamma(\tau)=$ $\exp \left[\mathrm{i} \hat{\mathcal{H}}_{\text {sys }} \tau\right] \hat{\Gamma} \exp \left[-\mathrm{i} \hat{\mathcal{H}}_{\text {sys }} \tau\right]$ and $\hat{x}(\tau)=\exp \left[\mathrm{i} \hat{\mathcal{H}}_{\text {env }} \tau\right] \hat{x} \exp \left[-\mathrm{i} \hat{\mathcal{H}}_{\text {env }} \tau\right]$. Substituting this into Eq. A.4 we get the Bloch-Redfield master equation that we gave in Section 3

Finally, to see when the Born approximation is justified, we must estimate the higher-order contributions that we are neglecting. The higher order contributions to $\boldsymbol{\Sigma}\left(t ; t_{1}\right)$ take a similar form to the second-order ones, but have more factors of $\hat{\Gamma} \hat{x}$ acting to the left and right of the density-matrix. The times at which these interactions can occur are chosen such that that $\boldsymbol{\Sigma}\left(t ; t_{1}\right)$ is irreducible (as discussed above). A typical fourth order contribution (those in the second line of Fig. A1b) will go like $|\hat{\Gamma} \hat{x}|^{4} t_{\mathrm{m}}^{3}$, compared with the second-order terms that went like $|\hat{\Gamma} \hat{x}|^{2} t_{\mathrm{m}}$. It is justifiable to neglect the fourth-order while keeping the second-order, only if $|\hat{\Gamma} \hat{x}| t_{\mathrm{m}} \ll 1$. Physically the constraint that $|\hat{\Gamma} \hat{x}| t_{\mathrm{m}} \ll 1$ means that the Bloch-Redfield master equation applies to situations where the decay rate of memory effects, $t_{\mathrm{m}}^{-1}$, is much faster than dissipative (relaxation and decoherence) rates $\sim|\hat{\Gamma} \hat{x}|^{2} t_{\mathrm{m}}$. There is no constraint on the ratio of

dissipative rates to the system's energy-scales, so the Bloch-Redfield equation can be applicable to strong (over-damped) and weak (under-damped) dissipation.

\section{Appendix B. A simple picture of initial-slips}

To understand how initial-slips work [16, 17, 18, 26, 23, 19, 24, 20, 21, 25], it helpful to neglect the matrix structure of the master equation. Then one has

$$
(\mathrm{d} / \mathrm{d} t) \rho(t)=-F(t) \rho(t)
$$


where $F(t)$ is time-dependent, but saturates at a finite value, $f_{\infty}$, for times greater than the memory time ( $F$ and $\rho$ are now numbers not matrices). This is traditionally approximated by [1, 2]

$$
(\mathrm{d} / \mathrm{d} t) \rho(t)=-f_{\infty} \rho(t) .
$$

Eq. (2.2) gives the wrong evolution for any initial condition, $\rho(0)$, because it is not justified for times less than the memory time. However, by multiplying $\rho(0)$ by an initial-slip one can ensure the evolution under the incorrect Eq. 2.2 coincides with the evolution under the correct Eq. 2.1) for all times much greater than the memory time. For the above equations, the initial-slip is simply exp $\left[-\int_{0}^{t} d t^{\prime}\left[F\left(t^{\prime}\right)-f_{\infty}\right]\right]$. For $t$ much greater than the memory time, $t_{\mathrm{m}}$, the initial-slip becomes time-independent (one can take the integral's upper-limit to $\infty$ since $\left[F\left(t^{\prime}\right)-f_{\infty}\right] \sim 0$ for $t^{\prime} \gg t_{\mathrm{m}}$ ). Thus one can take $\rho(0)$, "slip it" so that it becomes $\exp \left[-\int_{0}^{\infty} d t^{\prime}\left[F\left(t^{\prime}\right)-f_{\infty}\right]\right] \rho(0)$, and use that as the initial condition for evolution under the incorrect Eq. (2.2). The resulting $\rho(t)$ coincides with the correct result for all times much greater than $t_{\mathrm{m}}$, but will be absolutely meaningless for all times of order $t_{\mathrm{m}}$. Qualitatively the same analysis applies to the full master equation, but it is complicated by the matrix structure of the master equation (see e.g. Ref. 24]).

The above sketch of the initial-slip method, makes it clear that it is not suited to our analysis of positivity. The short-time dynamics (on timescales of order the memory time) that it generates are fictitious; a sudden initial-slip of the density matrix followed by evolution under an incorrect master equation. Studying the positivity for these fictitious short-time dynamics tells us nothing about whether the true short-time dynamics preserves positivity or not.

[1] Cohen-Tannoudji C, Dupont-Roc J and Grynberg G 1992 Atom-photon interactions (Wiley, New York)

[2] Breuer H-P and Petruccione F, 2002 The theory of open quantum systems (Oxford University Press, Oxford). A very good text on the subject, however their derivation of the (Bloch-) Redfield equation involves an assumption not made by Redfield. Redfield (working in the interaction picture) replaced $\hat{\rho}(s)$ with $\mathrm{e}^{\mathrm{i} \hat{\mathcal{H}}_{\mathrm{sys}}(s-t)} \hat{\rho}(t) \mathrm{e}^{-\mathrm{i} \hat{\mathcal{H}}_{\text {sys }}(s-t)}$ not $\hat{\rho}(t)$. The difference between these is that between $K_{1}$ and $K_{2}$ in Ref. 14. Our Appendix A follows Redfield.

[3] Nakamura Y, Pashkin Yu A and Tsai J S 1999 Nature 398 786. Vion D, Aassime A, Cottet A, Joyez P, Pothier H, Urbina C, Esteve D and Devoret M H 2002 Science 296 886. Chiorescu I, Nakamura Y, Harmans C J P M and Mooij J E 2003 Science 299 1869. Wallraff A, Schuster D I, Blais A, Frunzio L, Huang R-S, Majer J, Kumar S, Girvin S M and Schoelkopf R J 2004 Nature 431162.

[4] Engel H-A, Kouwenhoven L P, Loss D and Marcus C M 2004 Quantum Information Processing 3115 (Preprint cond-mat/0409294)

[5] Anderlini M, Lee P J, Brown B L, Sebby-Strabley J, Phillips W D and Porto J V 2007 Nature 448452

[6] Hall M 2008, preprint arXiv:0802.0606

[7] Lindblad G 1976 Commun. Math. Phys. 48119

[8] Alicki R and Lendi K 1987 Quantum dynamical semigroups and applications, vol 286 of Lecture notes in physics (Springer, Berlin).

[9] Bloch F 1957 Phys. Rev. 1051206

[10] Redfield A G 1957 IBM J. Res. Dev. 119

[11] Nakajima S 1958 Progr. Theor. Phys. 20948

[12] Zwanzig R 1960 J. Chem. Phys. 331338

[13] Schoeller H and Schön G 1994 Phys. Rev. B 5018436

[14] Dumcke R and Spohn H Z 1979 Z. Phys. B 34 419. Their $K_{2}$ is equivalent to the Bloch-Redfield equation, while their $K_{3}$ is a rotating-wave approximation of Bloch-Redfield.

[15] Spohn H Z 1980 Rev. Mod. Phys. 52 569, in particular Section V.

[16] Geigenmüller U, Titulaer U M and Felderhof B U 1983 Physica A 11941

[17] Haake F and Lewenstein M 1983 Phys. Rev. A 283606

[18] Haake F and Reibold R 1985 Phys. Rev. A 322462. 
Staying positive: ... perturbative master equations

[19] Gnutzmann S and Haake F, 1996 Z. Phys. B 101, 263

[20] Yu T, Diosi L, Gisin N, Strunz W T 2000 Phys. Lett. A265 331

[21] Maniscalco S, Intravaia F, Piilo J and Messina A 2004 J. Opt B: Quantum Semiclass. Opt. 6 S98 Preprint quant-ph/0306193

[22] Refs. 19 30 consider non-Lindblad master equations. In fact, manipulations of the type performed in Section 3.2 show that these equations do have the form in Eq. (1a) but do not satisfy Eq. [1b]. See Ref. [50] for a similar analysis of related works.

[23] Suárez A, Silbey R and Oppenheim I 1992 J. Chem. Phys. 975101

[24] Gaspard P and Nagaoka M 1999 J. Chem. Phys. 1115668

[25] Cheng Y C and Silbey R J 2005 J. Phys. Chem. B 10921399

[26] Gorini V, Verri M and Frigerio A 1989 Physica A 161, 357

[27] van Wonderen A J and Lendi K 2000 J. Stat.Phys. 100633

[28] In [20] there is an argument for the preservation of positivity for a damped harmonic oscillator. We believe that this argument is correct for infinitesimal times, but is not sufficient to prove positivity on timescales of order the memory time or longer. In [27] there is a general argument for the preservation of positivity, however we believe that this argument is only correct at infinite times. Ref. [27] also has an interesting discussion of conditions for positivity of a two-level system modelled (phenomenologically) by a Bloch equation with time dependent parameters. However in our experience, master equations with negative coupling constants do not take the form of Bloch equations (see the model in Section 6).

[29] Lidar D A, Bihary Z and Whaley K B 2001 Chemical Physics 26835

[30] Munro W J and Gardiner C W 1996 Phys. Rev. A 532633

[31] Wilkie J 2000 Phys. Rev. E 62 8808. Barnett S M and Stenholm S 2001 Phys. Rev. A 64033808. Budini A A 2004 Phys. Rev. A 69 042107. Daffer S, Wodkiewicz K, Cresser J D and McIver J K 2004 Phys. Rev. A $\mathbf{7 0} 010304$

[32] Shabani A and Lidar D A 2005 Phys. Rev. A 71 020101(R)

[33] Arguably the closest to a real qubit is, Budini A A and Schomerus H 2005 J. Phys. A: Math. Gen. 389251

[34] van Kampen N G 2004 J. Stat. Phys. 115 1057. van Kampen N G 2005 J. Phys. Chem. 109 21293.

[35] Pechukas P 1994 Phys. Rev. Lett. 73 1060. Alicki R 1995 Phys. Rev. Lett. 75, 3020. Pechukas P 1995 Phys. Rev. Lett. 75, 3021.

[36] Shaji A and Sudarshan E C G 2005 Physics Letters A 341, 48.

[37] As an example of a set of trace-class operators, consider a two-level system. One of many such sets of trace-class operators could be the following four operators $\hat{L}_{0}=\hat{\sigma}_{0} / \sqrt{2}, \hat{L}_{1}=\hat{\sigma}_{x} / \sqrt{2}$, $\hat{L}_{2}=\hat{\sigma}_{y} / \sqrt{2}$, and $\hat{L}_{3}=\hat{\sigma}_{z} / \sqrt{2}$, where $\sqrt{2}$ is for normalization. Yet there is nothing unique in this choice, and there is no requirement for Hermiticity, so another possible choice could have $\hat{L}_{1}=\hat{\sigma}_{+}$and $\hat{L}_{2}=\hat{\sigma}_{-}$, with $\hat{L}_{0}$ and $\hat{L}_{z}$ still as above. In any dissipative system the relevant basis of trace-class operators is given by the coupling to the environment.

[38] Some authors claim that factorized states are only relevant to those extremely rare situations in which the system-environment coupling is switched-on at $t=0$ (i.e. no coupling for all $t<0$ ). This is incorrect. Even with finite coupling for all $t$ (including $t<0$ ), a projective measurement on the system at $t=0$ prepares a given system state (with the experimenter knowing with certainty what this state is). This forces the total density matrix to be $\hat{\rho}(t=0) \otimes \hat{\rho}_{\text {env }}$, where $\hat{\rho}(t=0)$ is the given (pure) system state.

[39] Caldeira AO and Leggett AJ 1983 Ann. Phys. (NY) 149 374. Leggett AJ, Chakravarty S, Dorsey AT, Fisher MPA, Garg A and Zwerger W 1987. Rev. Mod. Phys. 591.

[40] Prokof'ev N and Stamp P 2000 Rep. Prog. Phys. 63669

[41] Makhlin Y, Schön G and Shnirman A 2003 Dissipation in Josephson qubits New Directions in Mesoscopic Physics (Towards Nanoscience), Fazio R, Gantmakher V F, and Imry Y (Eds) (Kluwer, Dordrecht) (Preprint cond-mat/0309049)

[42] This relationship between $S(\omega)$ and $A(\omega)$ can be shown as follows. At thermal equilibrium $\hat{\rho}_{\text {env }}=\sum_{n} P_{n}|n\rangle\langle n|$ where $|n\rangle$ is an eigenstate of $\hat{\mathcal{H}}_{\text {env }}$, and $P_{n} \propto \exp \left[-E_{n} / k_{\mathrm{B}} T\right]$. In this case $S(\omega)$ and $A(\omega)$ are $\propto \sum_{n m} P_{n}\left|x_{m n}\right|^{2}\left[\delta\left(E_{m}-E_{n}-\omega\right) \pm \delta\left(E_{m}-E_{n}+\omega\right)\right]$, where $+(-)$ is for $S(\omega)(A(\omega))$. Using $\sum_{n}=\int \mathrm{d} E^{\prime} \nu\left(E^{\prime}\right)$ and then writing energy as the average and difference of $E_{m}$ and $E_{n}$, we find $S(\omega)$ and $A(\omega)$ are $\propto \mathrm{e}^{\omega / 2 k_{\mathrm{B}} T} \pm \mathrm{e}^{-\omega / 2 k_{\mathrm{B}} T}$.

[43] see chapter VI of Landau L D and Lifshitz E M 1974 Quantum Mechanics (Pergamon,Oxford)

[44] The rotating-wave approximation fails if the dephasing rate is faster than $\left(E_{i^{\prime}}-E_{j^{\prime}}-E_{i}+E_{j^{\prime}}\right)$ with $i^{\prime} \neq i$ and $j^{\prime} \neq j$. Thus it always fails if there are (i) degeneracies (i.e. $E_{i^{\prime}}=E_{i}$ ), or (ii) two gaps of equal magnitude (i.e. $E_{i^{\prime}}-E_{j^{\prime}}=E_{i}-E_{j}$ ) [49]. 
[45] The effective Hamiltonian, $\hat{\mathcal{H}}_{\text {sys }}^{\prime}=\hat{\mathcal{H}}_{\text {sys }}-\frac{1}{2} \sum_{i j} \tilde{h}_{i j} \hat{P}_{i}^{\dagger} \hat{P}_{j}$ with $\tilde{h}_{i j}=\mathrm{i} \operatorname{tr}\left[\hat{\Gamma}^{\dagger} \hat{P}_{i}\right] \operatorname{tr}\left[\hat{P}_{j}^{\dagger} \hat{\Xi}\right]-$ $\mathrm{i} \operatorname{tr}\left[\hat{\Xi}^{\dagger} \hat{P}_{i}\right] \operatorname{tr}\left[\hat{P}_{j}^{\dagger} \hat{\Gamma}\right]$. Hence $\hat{\mathcal{H}}_{\text {sys }}^{\prime}=\hat{\mathcal{H}}_{\text {sys }}-\frac{1}{2} \sum_{i j} h_{i j}^{\prime} \hat{L}_{i}^{\dagger} \hat{L}_{j}$ where $h_{i j}^{\prime}=\left[\mathcal{U} \tilde{\mathbf{h}} u^{\dagger}\right]_{i j}$.

[46] Consider a 3-level system whose density matrix is diagonal with the three following elements; $\rho_{11}=(1+x) / 3, \rho_{22}=(1-x) / 3, \rho_{33}=1 / 3$. For $1<x \leq \sqrt{3}$, this density-matrix is unphysical because it is not positive (the probability $\rho_{22}<0$ ), yet the purity $P \leq 1$.

[47] This is easily shown by writing $s_{x}, s_{y}, s_{z}$ in polar coordinates, and showing that the maxima of the resulting function are at $(\theta, \phi)=\left(\pi / 2, \frac{1}{2} B t\right)$ and $(\theta, \phi)=\left(\pi / 2, \pi+\frac{1}{2} B t\right)$.

[48] Since we approximate $\mathbb{K}$ by $\mathbb{K}^{\text {sys }}$, and not by 1 , our derivation is equivalent to Redfield's [10], and not the summary of Redfield's derivation in Ref. 2 .

[49] Clusel $\mathrm{M}$ and Ziman T 2007 Preprint arXiv:0705.1631 Clusel M and Ziman T 2008 in preparation.

[50] Isar A, Sandulescu A, Scutaru H, Stefanescu E and Scheid W 1994 Int. J. Mod. Phys. E 3, 635 (see in particular Section 3) 